\title{
Entropy Analysis in Relativistic Heavy-Ion Collisions
}

\author{
Shakeel Ahmad, ${ }^{1}$ A. Ahmad, ${ }^{2}$ Anuj Chandra, ${ }^{1}$ M. Zafar, ${ }^{1}$ and M. Irfan ${ }^{1}$ \\ ${ }^{1}$ Department of Physics, Aligarh Muslim University, Aligarh 202002, India \\ ${ }^{2}$ Department of Applied Physics, Aligarh Muslim University, Aligarh 202002, India
}

Correspondence should be addressed to Shakeel Ahmad; shakeel.ahmad@cern.ch

Received 2 June 2013; Revised 16 August 2013; Accepted 18 August 2013

Academic Editor: Fu-Hu Liu

Copyright (C) 2013 Shakeel Ahmad et al. This is an open access article distributed under the Creative Commons Attribution License, which permits unrestricted use, distribution, and reproduction in any medium, provided the original work is properly cited.

\begin{abstract}
Entropy creation in multiparticle system is investigated by analysing the experimental data on ion-ion collisions at AGS and SPS energies and the results thus obtained are compared with those predicted by multiphase transport and correlation-free Monte Carlo models. Entropies produced in limited- and full-phase space are observed to increase with increasing beam energy. The entropy values, normalized to the maximum rapidity and plotted against pseudorapidity (bin width also normalized to the maximum rapidity), are found to be energy independent, exhibiting a kind of entropy scaling. Such scaling is observed in the full phase space as well as in the regions confined to the forward or backward hemispheres. The findings also reveal that there exist strong correlations amongst the particles produced in the forward and backward hemispheres around the midrapidity. These correlations are found to be of short range in nature, and the contributions from the long-range correlations seem to be absent. PACS numbers: 25.75-q, 25.75.Gz.
\end{abstract}

\section{Introduction}

One of the main goals of studying nucleus-nucleus (AA) collisions at relativistic energies is to study the properties of strongly interacting matter under extreme conditions of initial energy density and temperature, where formation of quark-gluon-plasma (QGP) is envisaged to take place [1, 2]. Fluctuations in the physical observables in relativistic AA collisions are regarded as one of the important signals for QGP formation because of the idea that in many body systems, phase transition may cause significant changes in the quantum of fluctuations of an observable from its average behaviour [2,3]. For example, when a system undergoes a phase transition, heat capacity changes abruptly, whereas the energy density remains a smooth function of temperature [3]. Entropy is regarded as the most significant characteristic of a system having many degrees of freedom [4-7]. Processes in which particles are produced may be regarded as the socalled dynamical systems [4-8] in which entropy is generally produced. Investigations involving the local entropy produced in relativistic AA collisions are expected to provide direct information about the internal degrees of freedom of the QGP medium and its evolution [9]. It has been suggested $[4-7,9-12]$ that the event coincidence probability method of measuring entropy proposed by Ma [13-15] is well suited for the analysis of local properties in multiparticle systems produced in high energy collisions. This method is applicable to both hadron-hadron and AA collisions $[9,11]$. In AA collisions, entropy measurement can be used not only to search for QGP formation but it may also serve as an additional tool to investigate the correlations and event-byevent fluctuations [4-7, 9]. Analysis of the experimental data on hh collisions over a wide range of incident energy (up to $\sqrt{s}=900 \mathrm{GeV}$ ) carried out by Simak et al. [16] indicates that entropy increases with beam energy, while the entropy per unit rapidity seems to be an energy independent quantity. These findings indicate the presence of ultimate scaling over an energy range extending up to a few TeV. Such scaling has also been observed in pp collisions at LHC energies [17]. For AA collisions, however, only a few attempts have been made [18-21] to study the entropy production in multiparticle systems. It was, therefore, considered worthwhile to carry out a well-focused study of entropy production and subsequent scaling in AA collisions by analysing the experimental data over a wide range of incident energies. The findings are compared with the predictions of a multiphase transport 
(AMPT) and an independent emissions hypothesis (IEH) models.

\section{Method of Analysis}

In high energy AA collisions, as the two colliding nuclei interpenetrate, the collision between the participating nucleons causes the combined system to fly apart. Initially, there would be a large random component to the particles' velocities, which is described by temperature [22]. As the particles move outwards, their velocity vectors become more and more oriented in radial direction with a reduced random component [22]. In thermodynamic terms, the nuclear gas cools, with thermal energy converted to collective flow energy in the radial direction. As the time elapses, volume of the system, $V$, increases, whereas the temperature, $T$, decreases. At a certain point of time, collisions between participating nucleons cease and the measurable characteristics of the system freeze out [22], but the thermodynamic analysis of the system in terms of $V$ and $T$ is very difficult as both the quantities change rapidly with time. It has, however, been suggested by Seimens and Kapusta [23] that instead of studying $T$ and $V$, entropy may easily be studied as it grows rapidly only during the initial stage of the collision and does not significantly change in the later stage, when the system expands and cools. Since, in heavy-ion collision experiments, measurements are confined only to the final state particles, which are mostly hadrons, net entropy is, therefore, expected to provide invaluable insight into the state of matter the in early stage of the collision, as it is nearly conserved between initial thermalization and freezeout [24]. After freeze-out, when particles freely stream out, entropy remains essentially constant. Entropy may increase only because of viscous effects, shock waves, and decoupling processes. The entropy from the final state, thus, provides and an upper bound for the entropy of the initial state.

Entropy of produced particles is calculated from their multiplicity distribution using [20]:

$$
S=-\sum_{n} P_{n} \ln P_{n}
$$

where $P_{n}$ is the probability of $n$ relativistic charged particles being produced in an interaction. If there are $\nu$-independent sources which contribute to the particle production, the entropy being an additive quantity may be expressed as [20]:

$$
S=-\sum_{i=1}^{v} S_{i}
$$

The invariance of entropy under an arbitrary change of multiplicity scale allows to choose a subsample of particles, like charged particles. The entropy of the emitted relativistic charged particles is, therefore, calculated for the experimental and simulated data sets selected in the present study.

\section{Details of the Data}

A stack of G5 emulsion, horizontally exposed to $14.5 \mathrm{~A} \mathrm{GeV} / \mathrm{c}$

${ }^{28} \mathrm{Si}$ ions from AGS at BNL, is used. The events were searched for by following an along the track method and the ones which satisfy the following criteria were selected for carrying out various measurements and analysis:

(i) the tracks of the incident particles producing events should not be inclined more than $3^{\circ}$ with respect to the mean beam direction and

(ii) the events should lie at a depth $\geq 20 \mu \mathrm{m}$ from the top or the bottom surface of the emulsion pellicle.

Adopting the above criteria, a sample consisting of 1039 interactions characterized by $N_{h} \geq 0$ were collected; $N_{h}$ denotes the number of tracks with ionization, $I \geq 1.4 I_{0}$, $I_{0}$ is the minimum ionization produced by a singly charged relativistic particle. From this sample, events produced due to the $\mathrm{AgBr}$ group of target were sorted out on the basis of their $N_{h}$ values. Events with $N_{h} \geq 8$ are envisaged to be produced exclusively due to the interactions with $\mathrm{AgBr}$ targets, whereas those having $N_{h} \leq 7$ are produced either due to the interactions with $\mathrm{H}$ or $\mathrm{CNO}$ group of nuclei or due to peripheral collisions with $\mathrm{AgBr}$ nuclei [25-31]. Following these criteria, 561 events due to $\mathrm{AgBr}$ targets were selected for the present analysis. Furthermore, two other sets of data on the interactions of ${ }^{16} \mathrm{O}$ ions with $\mathrm{AgBr}$ targets at 60 and $200 \mathrm{~A} \mathrm{GeV} / \mathrm{c}$, from the emulsion experiment performed by EMU01 collaboration [25-28], are also analysed; number of events in these data sets are 422 and 223 , respectively. It should be emphasized that the conventional emulsion technique has two main advantages over the other detectors: (i) its $4 \pi$ solid angle coverage and (ii) emulsion data are free from biases due to full phase space coverage. In the case of other detectors, only a fraction of charged particles are recorded due to limited acceptance cones. This not only reduces the charged particle multiplicity but also distorts some of the event characteristics, such as particle density fluctuations [32]. Emission angle, $\theta$, and azimuthal angle, $\phi$, were measured for each track produced by relativistic charged particles with respect to the mean beam direction. Using the measured values of $\theta \mathrm{s}$, pseudorapidity variable, $\eta$ of each of the produced relativistic charged particles is estimated using the relation $\eta=-\ln \tan (\theta / 2)$. The number of relativistic charged particles in a selected $\eta$ window is, thus, counted to estimate the probability of producing $n$ charged particles, $P_{n}(\Delta \eta)$ for the entire data sample. $P_{n}(\Delta \eta)$ values, thus obtained, were used to determine the entropy produced in a particular $\eta$-window using (1), as described in the next section. In order to compare the findings of the present study with the predictions of Monte Carlo model and AMPT [33], event samples matching the real data are simulated using the code ampt-v-1.2.21. The number of events in each simulated data set is equal to that in the real data sample. The events are simulated by taking into account the percentage of interactions which occur in the interactions of projectile with various target nuclei in emulsion [29]. The values of impact parameter for each data set is so set that the mean multiplicities of relativistic charged particles become nearly equal to those obtained for the real data sets. 


\section{Results and Discussion}

Probability, $P_{n}(\Delta \eta)$, of producing $n$ relativistic charged particles in a pseudorapidity window of fixed width is calculated by selecting a window of fixed width, $\Delta \eta=0.5$. This window is chosen so that its midposition coincides with the centre of symmetry of $\eta$ distribution, $\eta_{c}$. Thus, all the relativistic charged particles having their $\eta$ values lying in the interval $\eta_{c}-(\Delta \eta / 2) \leq \eta \leq \eta_{c}+(\Delta \eta / 2)$ are counted to evaluate $P_{n}$. The window width is then increased in a step of 0.5 until a region $\eta_{c} \pm 3.0$ is covered. The values of entropy for different $\eta$ windows, ranging from 0.5 to 6.0 , is then calculated using (1). Variations of entropy, $S$, with $\Delta \eta$ for the real and AMPT data samples are displayed in Figure 1. It may be noted from the figure that with increasing $\Delta \eta$, entropy first increases rather quickly then slows down and thereafter saturates beyond $\Delta \eta \sim 4$ 4. Such a trend is expected because of the fact that with increasing $\Delta \eta$, particle multiplicity increases, first rapidly and then slowly, causing entropy to increase in a similar fashion. However, beyond $\Delta \eta \sim 4.0$, multiplicity increases nominally yielding essentially the same value of entropy. It may also be noted from the figure that for a given $\Delta \eta$, entropy increases with increasing beam energy. Furthermore, a comparison of the plots for the real and AMPT simulated events, shown in Figure 1, shows that the entropy values for the experimental data are close to those predicted by the AMPT model. Studying the multiplicity dependence of average phase space densities and entropies of thermal pions, Sinyukov and Akkelin [34] have reported the presence of deconfinement and chiral phase transitions in AA collisions at relativistic energies. The observed energy dependence of entropy per unit rapidity by these workers have been interpreted as the chemically equilibrium pion number which is frozen at initially very high temperature that increases with collision energy [34] with regard to the experimental scenario, entropy evolution has been investigated by Simak et al. [16] in hh collisions in the energy range of $\sqrt{s}=22$ to $900 \mathrm{GeV}$. Entropy increase has been observed [16] with increasing projectile energy in the entire energy range considered. Similar energy dependence of entropy has also been observed by Mizoguchi and Biajima [17] at LHC energies $(\sqrt{s}=0.2$ to $7 \mathrm{TeV})$.

To check whether the observed entropy behaviour is a distinct feature of the data or arises solely due to fluctuations in the event multiplicities, correlation-free Monte Carlo events are generated and analysed. These events are generated in the frame work of Independent emission hypotheses (IEH) by adopting the following criteria $[30,31,35,36]$.

(1) Multiplicity distribution of the produced particles should be similar to the one obtained for the experimental data.

(2) There should be no correlation between the produced particles.

(3) For each event, single particle inclusive distribution in $\eta$ space is set to have Gaussian shape with its mean value, $\langle\eta\rangle$, and dispersion, $\sigma$, equal to their corresponding values for the real event.

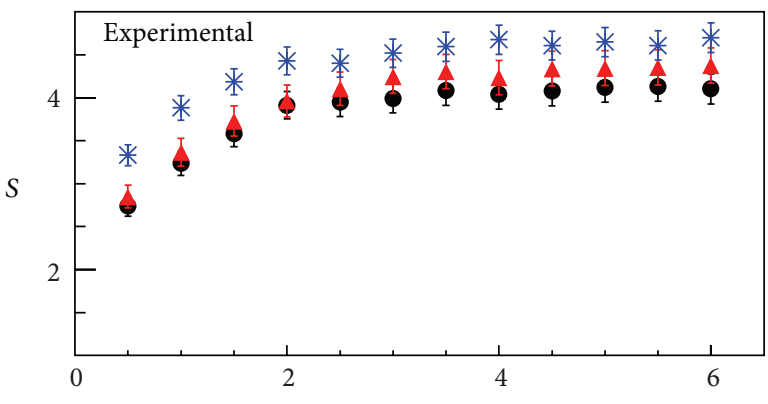

$\Delta \eta$

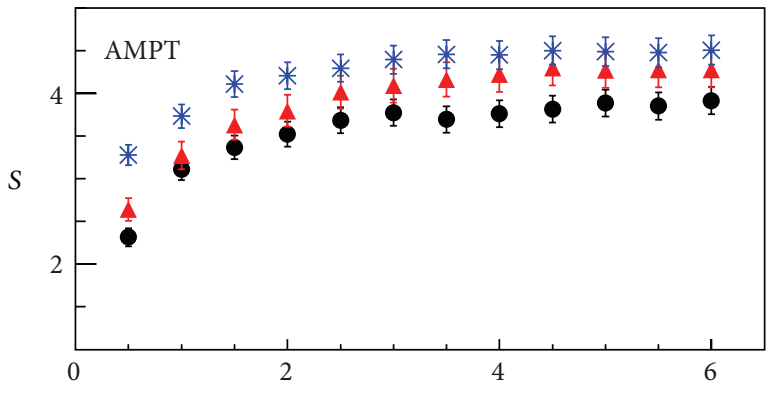

$\Delta \eta$

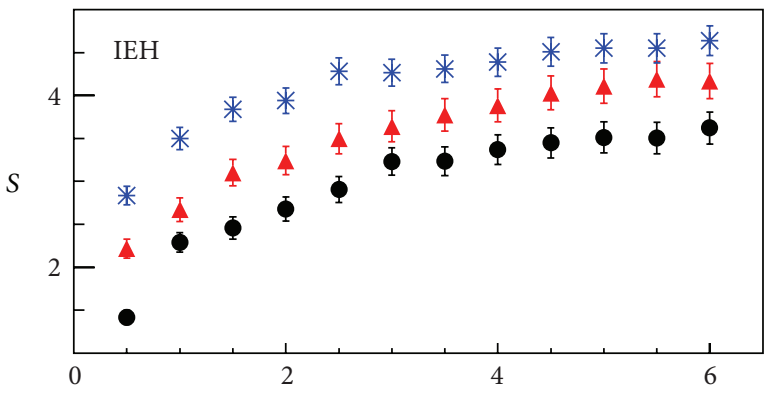

$\Delta \eta$

$$
\begin{aligned}
& \text { - } 14.5 \mathrm{~A} \mathrm{GeV} / \mathrm{c}^{28} \mathrm{Si}-\mathrm{AgBr} \\
& \text { * } 60 \mathrm{~A} \mathrm{GeV} / \mathrm{c}^{16} \mathrm{O}-\mathrm{AgBr} \\
& \text { * } 200 \mathrm{~A} \mathrm{GeV} / \mathrm{c}^{16} \mathrm{O}-\mathrm{AgBr}
\end{aligned}
$$

FIGURE 1: Variations of entropy with $\eta$-bin width for the experimental, AMPT, and IEH events at various energies.

By applying the above criteria, three sets of correlation-free Monte Carlo (IEH) events corresponding to the three real data samples are simulated and analysed. The number of events in each sample is equal to those for the real data. Variations of $S$ with $\Delta \eta$ for these events at the three incident energies considered are shown in Figure 1 (bottom panel). It is evident from the figure that the trend of variation of $S$ with $\Delta \eta$ for the IEH events is markedly different from those observed for the real and the AMPT data. This indicates that the observed entropy dependence on the width of $\eta$ window is a distinct feature of the data and definitely not a manifestation of statistics. These findings are, thus, in fine agreement with those reported earlier [16] for hh collisions in the energy range from $\sim 2 \mathrm{GeV}$ to a few $\mathrm{TeV}$. In AA collisions too, entropy has been observed by Khan et al. [21] to increase with increasing beam energy.

It has been reported [16] that for hh collisions in the energy range of $\sim 22$ to $900 \mathrm{GeV}$, total entropy produced in 
a limited pseudorapidity bin, when normalized to maximum rapidity in the centre-of-mass frame, is found to be essentially independent of the energy and identity of the colliding hadrons, indicating, thereby, the presence of a kind of entropy scaling. Such a scaling of entropy has also been observed by Mizoguchi and Biajima [17] in pp collisions at $\mathrm{cms}$ energy range, $0.2-7 \mathrm{TeV}$. In case of AA collisions too, a similar scaling behaviour has been observed by Khan et al. [21] in 4.5 and $14.5 \mathrm{~A} \mathrm{GeV} / \mathrm{c}^{28} \mathrm{Si}$-nucleus collisions and also by Ghosh et al. [20] in $60 \mathrm{~A} \mathrm{GeV} / \mathrm{c}{ }^{16} \mathrm{O}-\mathrm{AgBr}$ and $200 \mathrm{~A} \mathrm{GeV} / \mathrm{c}^{32} \mathrm{~S}$ $\mathrm{AgBr}$ collisions. An attempt is, therefore, made to examine the occurrence of entropy scaling with the present data that would definitely cover a significant energy range (AGS and SPS). For this purpose, values of the maximum rapidity in the centre-of-mass frame, $Y_{m}$, are calculated using the relation [20]

$$
Y_{m}=\ln \left(\frac{\sqrt{s}}{m_{\pi}}\right),
$$

where $\sqrt{s}$ denotes the centre-of-mass energy of the participating system and $m_{\pi}$ represents the rest mass of a pion. The value of $\sqrt{s}$ is calculated from [37]

$$
\sqrt{s}=\sqrt{\left(N_{p} m\right)^{2}+\left(N_{t} m\right)^{2}+2 N_{t} m E_{\mathrm{beam}}}
$$

where $E_{\text {beam }}$ is the energy of projectile nucleus in laboratory frame, $N_{p}$ and $N_{t}$, respectively, denote the numbers of participating projectile and target nuclei, and $m$ is the nucleon mass. Values of $N_{p}$ and $N_{t}$ used for calculating $\sqrt{s}$ are the event-averaged values estimated from the AMPT event samples.

Variations of entropy normalized to maximum rapidity, $S / Y_{m}$ for the experimental, AMPT and IEH data are exhibited in Figure 2. It may be seen in the figure that for the experimental and AMPT data, $S / Y_{m}$ first increases up to $\Delta \eta / Y_{m} \sim 0.5$ and thereafter tends to acquire almost a constant value. It is interesting to point out that the data points corresponding to various energies overlap and fall on a single curve, indicating the presence of entropy scaling which is well supported by AMPT. No such scaling is, however, observed in the case of IEH data. This suggests that the observed scaling in the case of real data is truly of dynamical nature and the findings are in fine agreement with the AMPT predictions.

Investigations involving forward-backward (F-B) multiplicity fluctuations and correlations [30, 38-44] suggest that event-by-event (ebe) multiplicities of relativistic charged particles in the forward (F) and backward (B) hemispheres are not equal. This prompts one to study multiplicity fluctuations by comparing the ebe multiplicity, $n_{f}$, in a pseudorapidity window of width $\Delta \eta$ placed in the $\mathrm{F}$ region with the multiplicity $n_{b}$ observed in an identical window in the $\mathrm{B}$ region; the two windows are chosen in such a way that they are symmetric around $\eta_{c}$. Using these definitions, a number of attempts have been made to investigate F-B multiplicity correlations and fluctuations either by examining the dependence of $n_{f}$ on $\left\langle n_{b}\right\rangle$ [42-46] or by considering [30, 38-41] a multiplicity asymmetry variable $C=\left(n_{f}-n_{b}\right) / \sqrt{\left(n_{f}+n_{b}\right)}$. These
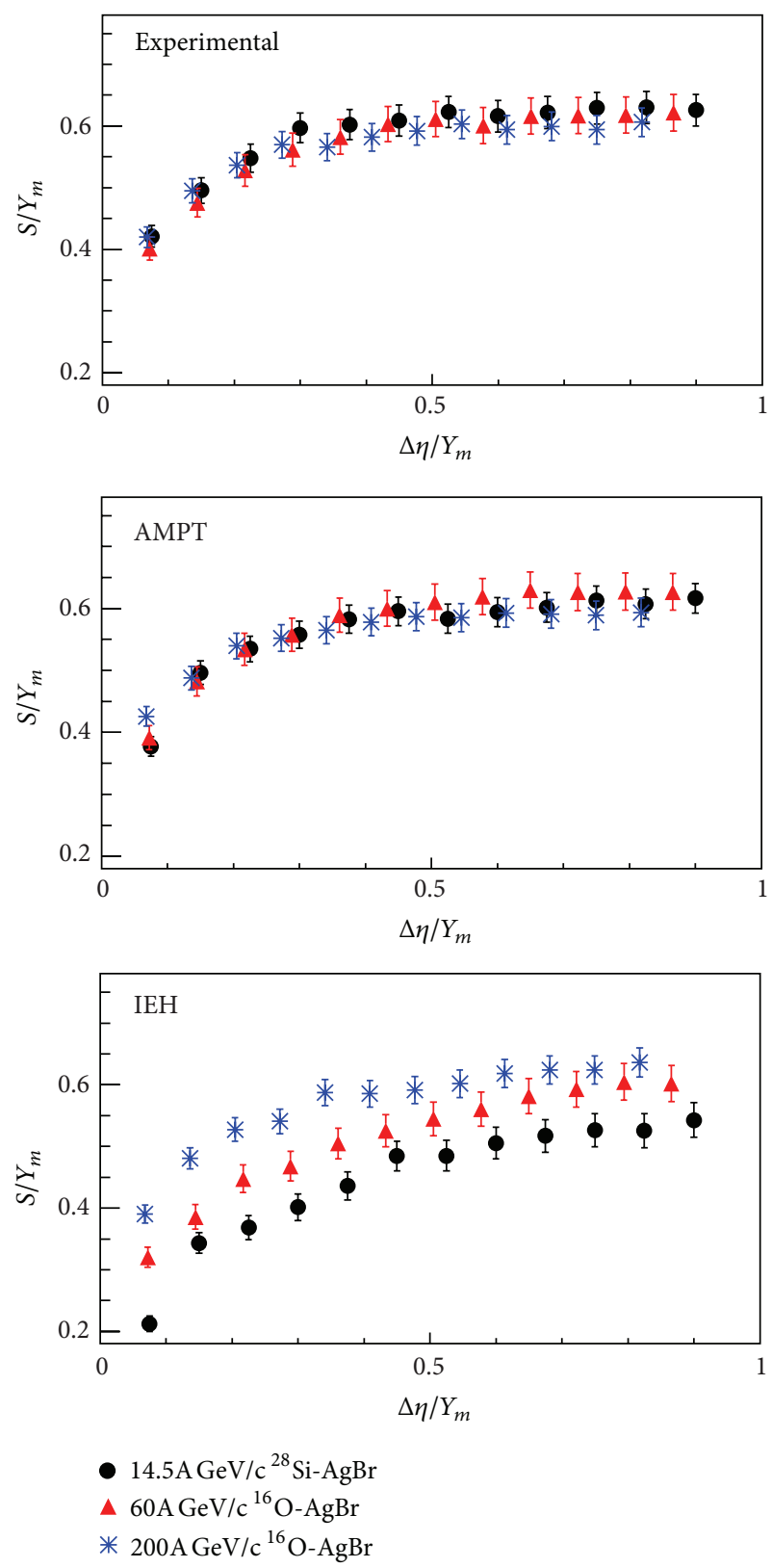

FIgure 2: Dependence of $S / Y_{m}$ on $\Delta \eta / Y_{m}$ for the real, AMPT, and IEH event samples at the three incident energies.

investigations observe presence of strong F-B correlations. Such correlations are believed to arise due to isotropic decays of cluster-like objects in $\mathrm{F}$ or $\mathrm{B} \eta$ region. The presence of ebe multiplicity asymmetry, thus, indicates that the value of the entropy in the two regions are different. In order to test this, we have studied the entropy production and its scaling separately in $\mathrm{F}$ and $\mathrm{B}$ regions by selecting a $\eta$ window of width, $\Delta \eta$, and placing it in $\mathrm{F}$ region such that all the charged particles having their $\eta$ values lying in the interval $\eta_{c} \leq \eta \leq$ $\eta_{c}+\Delta \eta$ are counted to estimate the entropy values in the $\mathrm{F}$ region. Similarly, the entropy values in the B region are estimated by picking up the charged particles with their $\eta$ values in the range $\eta_{c} \geq \eta \geq \eta_{c}-\Delta \eta$. Dependence of entropy on the $\eta$-bin width in $\mathrm{F}$ and $\mathrm{B}$ regions for the experimental 

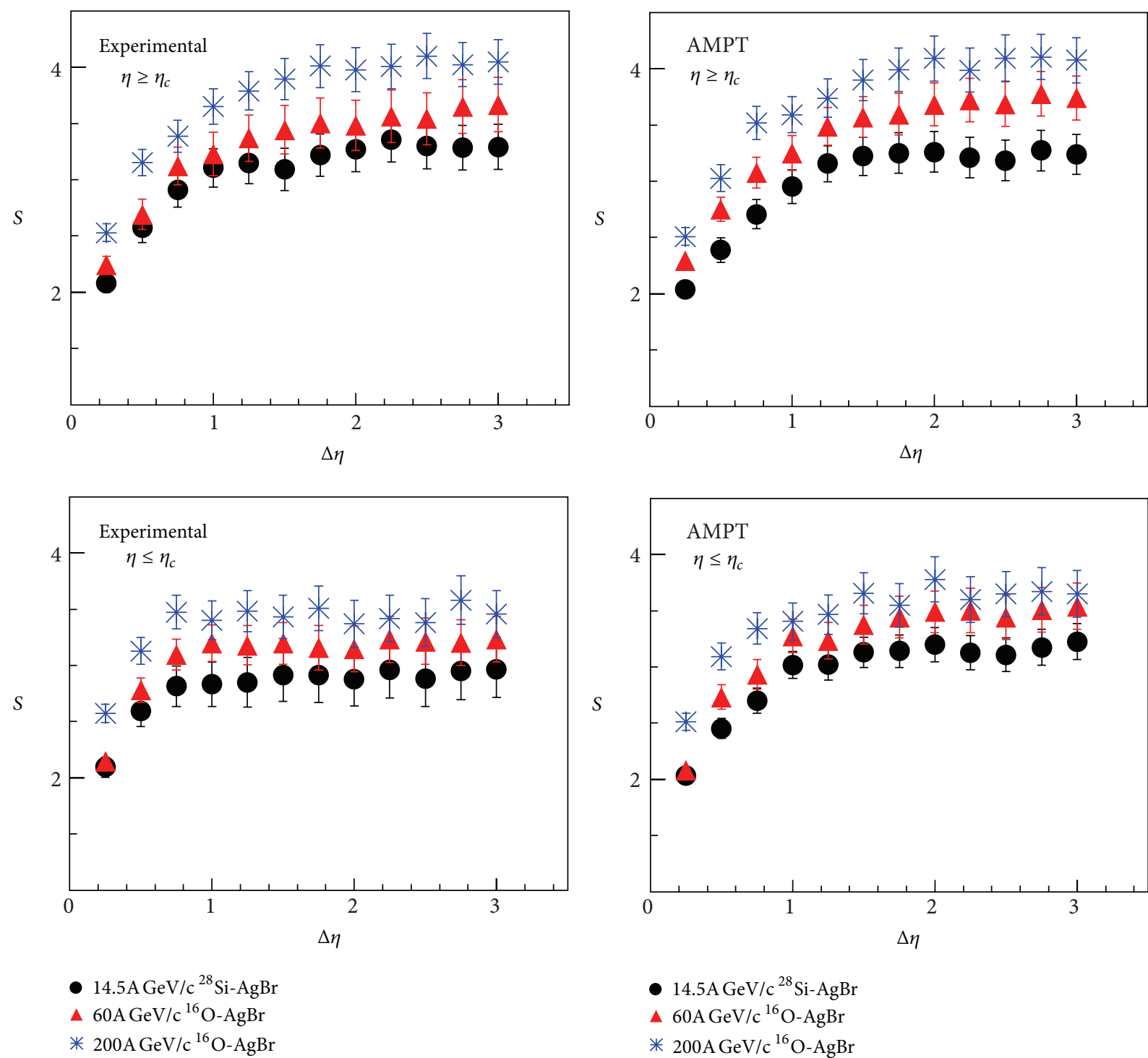

FIgURE 3: Variations of $S$ with $\Delta \eta$ for the experimental and AMPT events in forward and backward hemispheres.

and AMPT data are displayed in Figure 3. Similar plots for the IEH events are shown in Figure 4. Th following observations may be made from these figures.

(i) In the case of experimental and AMPT data, entropy increases with increasing $\Delta \eta$ up to $\sim 1.5$ and thereafter acquires nearly a constant value in both $\mathrm{F}$ and $\mathrm{B}$ hemispheres.

(ii) For a given $\Delta \eta$, entropy increases with increasing beam energy.

(iii) For IEH events, on the other hand, with increasing $\Delta \eta$, the value of $S$ first increases quickly then rather slowly but does not saturate. Thus, the trends of variations of $S$ with $\Delta \eta$ for the real and AMPT data are observed to be quite different from those observed for the IEH data.

(iv) It is interesting to note that for the IEH event samples, the values of $S$ against $\Delta \eta$ in $\mathrm{F}$ and $\mathrm{B}$ regions are nearly the same. For the real and AMPT data, on the other hand, $S$ acquires rather a larger value in the $\mathrm{F}$ hemisphere as compared to those for the B hemisphere. Such a difference in $S$ values is noticed in the entire $\Delta \eta$ region and for all the three energies considered.

The observed difference in the entropy values in the two regions for the experimental and AMPT events (and not with the IEH events) may arise due to the strong correlations existing between the particles belonging to the adjacent $\mathrm{F}$ and $B$ regions around midrapidity $[9,39-41]$. It is commonly believed [38-46] that the forward-backward correlations observed are of short-range type and there are almost no or very small contributions from the long-range correlations, particularly at lower energies. In order to ensure further that whether or not the observed entropy difference arises due to the particle correlations of short-range type, entropy values are calculated in $\mathrm{F}$ and $\mathrm{B}$ regions after leaving a gap of $2 \eta$ units 

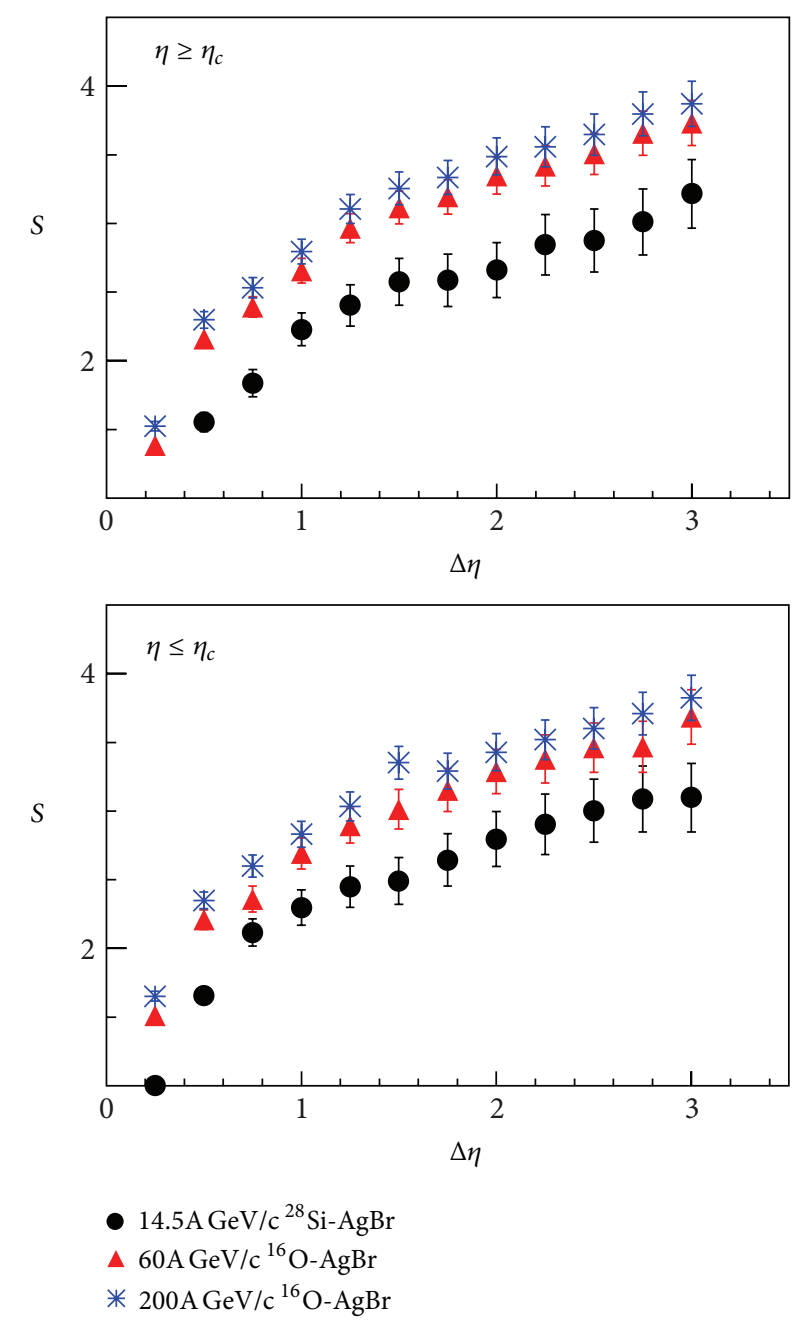

Figure 4: The same plots as in Figure 3 but for the IEH events.

between the two regions; that is, particles with $\eta \leq \eta_{c}+1.0$ in the $\mathrm{F}$ region and those with $\eta \geq \eta_{c}-1.0$ in the $\mathrm{B}$ regions are not counted. Dependence of $S$ on $\Delta \eta$ for such regions are displayed in Figures 5(a) and 5(b). Similar plots for an F-B separation of $3 \eta$ units are also shown in Figures 5(c) and 5(d). It is interesting to note in Figure 5 that the entropy difference in the two regions is much smaller as compared to those reflected from Figure 3. Moreover, for the $\mathrm{F}$ and $\mathrm{B}$ regions separated by $3 \eta$ units, this difference almost vanishes and the entropy values against $\Delta \eta$ in the two regions are nearly the same (Figure 5). Results from the analysis of the AMPT events also supports the experimental observations. These findings, thus, suggest that there exist strong correlations amongst the particles belonging to adjacent $\mathrm{F}$ and $\mathrm{B}$ regions and are mostly of short-range type. On introducing a separation between the two regions, the F-B correlations become weaker and finally vanish for larger separations, giving nearly the same entropy values for the two hemispheres.

In order to test whether the relativistic charged particles emitted in F and $\mathrm{B}$ hemispheres exhibit the same kind of entropy scaling as observed when the particles of the two regions are collectively considered, variations of $S / Y_{m}$ with
$\Delta \eta / Y_{m}$ for the charged particles emitted in $\mathrm{F}$ and $\mathrm{B}$ regions are examined for real, AMPT, and IEH data sets. These variations are displayed in Figure 6. It is clear from the figure that the data points corresponding to three energies overlap and indicate the presence of entropy scaling in both $\mathrm{F}$ and $\mathrm{B}$ regions. The observed scaling behaviour is nicely reproduced by the AMPT data sets. Moreover, the results for the IEH events confirm that the observed entropy scaling is a distinguishable feature of the data rather than a manifestation of statistics.

\section{Conclusions}

Based on the findings of the present work, the following significant conclusions may be reached.

(1) With widening of the $\eta$ windows, entropy first increases and thereafter acquires nearly a constant value.

(2) For a given $\Delta \eta$, entropy increases with increasing beam energy. 


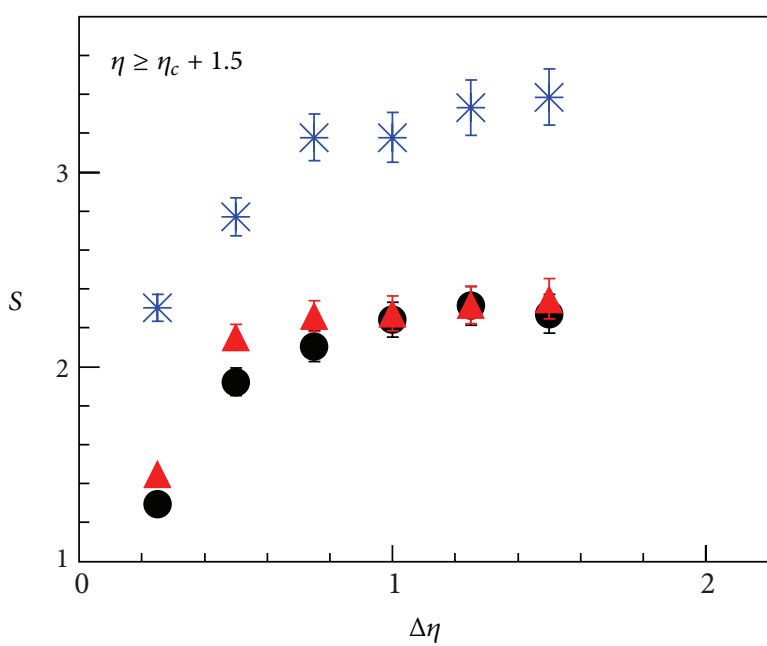

(a)

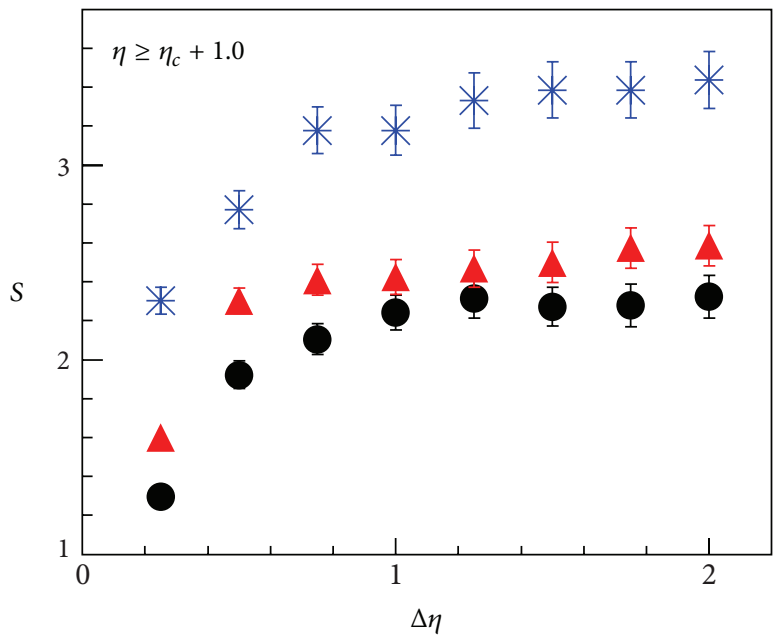

- $14.5 \mathrm{~A} \mathrm{GeV} / \mathrm{c}^{28} \mathrm{Si}-\mathrm{AgBr}$

- $60 \mathrm{~A} \mathrm{GeV} / \mathrm{c}^{16} \mathrm{O}-\mathrm{AgBr}$

* $200 \mathrm{~A} \mathrm{GeV} / \mathrm{c}^{16} \mathrm{O}-\mathrm{AgBr}$

(c)

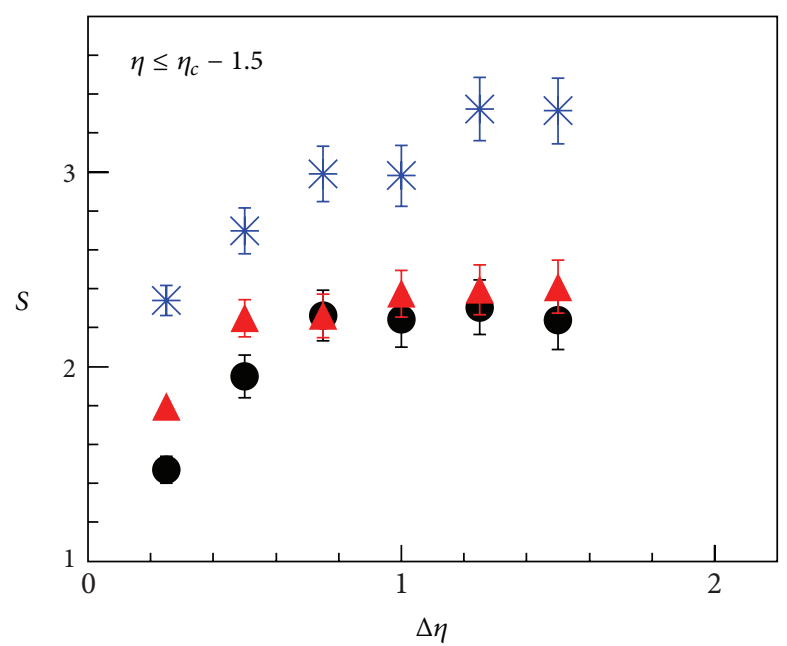

(b)

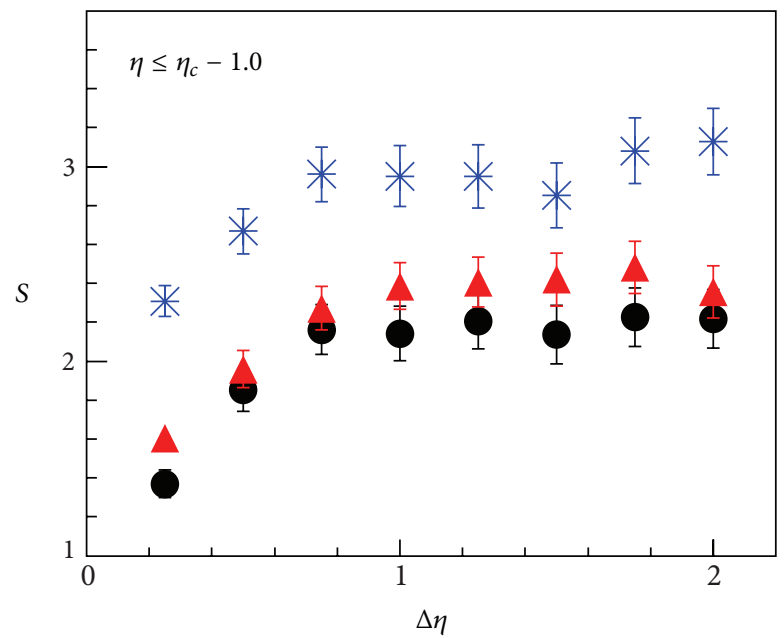

- $14.5 \mathrm{~A} \mathrm{GeV} / \mathrm{c}^{28} \mathrm{Si}-\mathrm{AgBr}$

$\triangle 60 \mathrm{~A} \mathrm{GeV} / \mathrm{c}^{16} \mathrm{O}-\mathrm{AgBr}$

* $200 \mathrm{~A} \mathrm{GeV} / \mathrm{c}^{16} \mathrm{O}-\mathrm{AgBr}$

(d)

FIGURE 5: $S$ versus $\Delta \eta$ plots for the forward and backward regions separated by $\eta$ gaps of 3 units ((a), (b)) and 2 units ((c), (d)).

(3) Dependence of $S / Y_{m}$ on $\Delta \eta / Y_{m}$ shows the presence of entropy scaling in AA collisions at AGS and SPS energies. Such a scaling is observed to hold good even if the particles emitted in the forward and backward regions are separately considered.

(4) Entropy dependence on pseudorapidity bin width, examined separately in forward and backward hemispheres, indicates the presence of strong correlations amongst the particles emitted in the two hemispheres around mid-pseudorapidity. On effecting a separation between the two hemispheres, these correlations are observed to become weaker and finally vanish when the separation between the two hemispheres becomes relatively large. This suggests that the observed correlations are of short range in nature, arising due to the "clusters" and the high mass states, produced during the initial stage of collisions, which finally decay isotropically in their centre-of-mass frame to real physical hadrons, while the contributions from the long-range correlations appear to be absent.

The findings of the present study reveal that entropy per unit rapidity increases with collision energy, whereas when normalized to maximum rapidity, becomes energy independent. These results are in fair agreement with those obtained from theoretical calculations of average phase space density and entropy in thermal hadronic system at the final (freezeout) stage of AA collisions, carried out by Sinyukov and Akkelin [34]. Theoretical investigation [24] of entropy per unit rapidity at freeze-out with minimal model dependence 


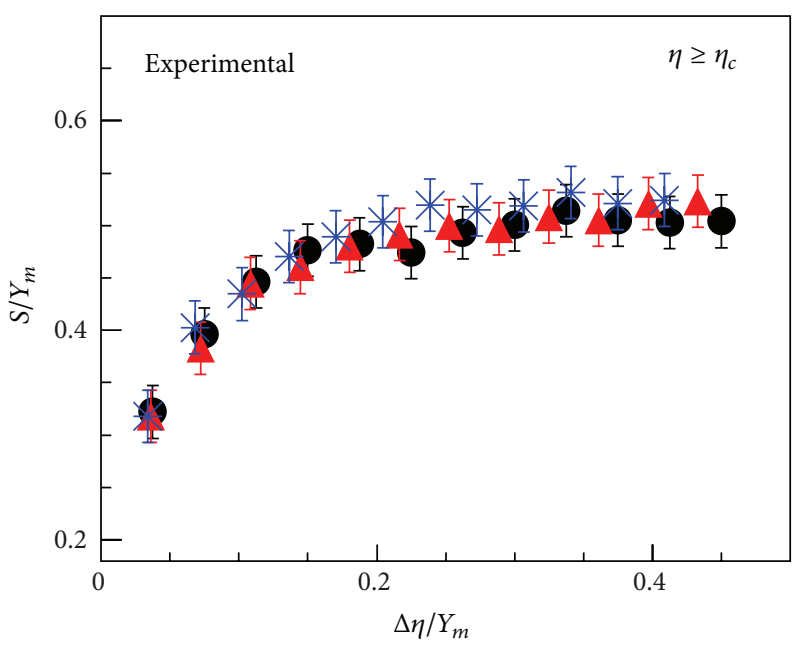

(a)

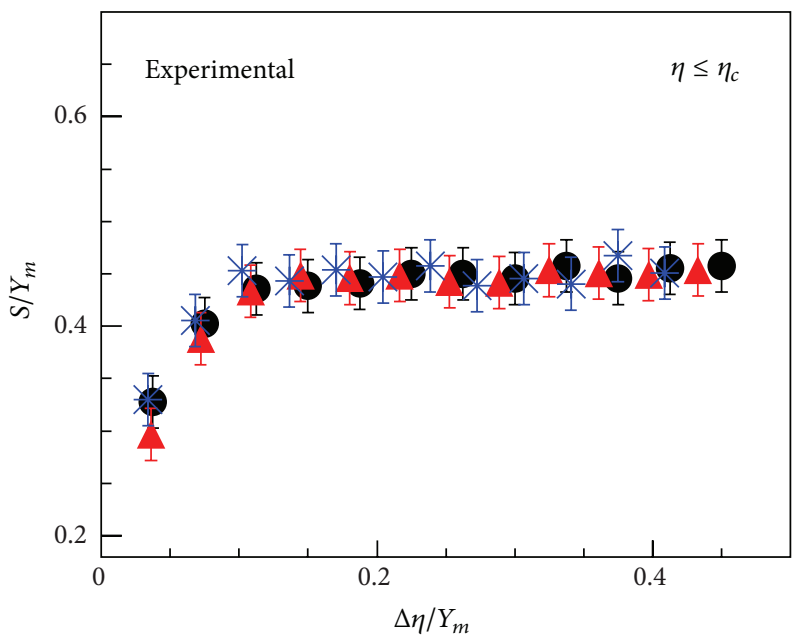

- $14.5 \mathrm{~A} \mathrm{GeV} / \mathrm{c}^{28} \mathrm{Si}-\mathrm{AgBr}$

- $60 \mathrm{AGeV} / \mathrm{c}^{16} \mathrm{O}-\mathrm{AgBr}$

* $200 \mathrm{~A} \mathrm{GeV} / \mathrm{c}^{16} \mathrm{O}-\mathrm{AgBr}$

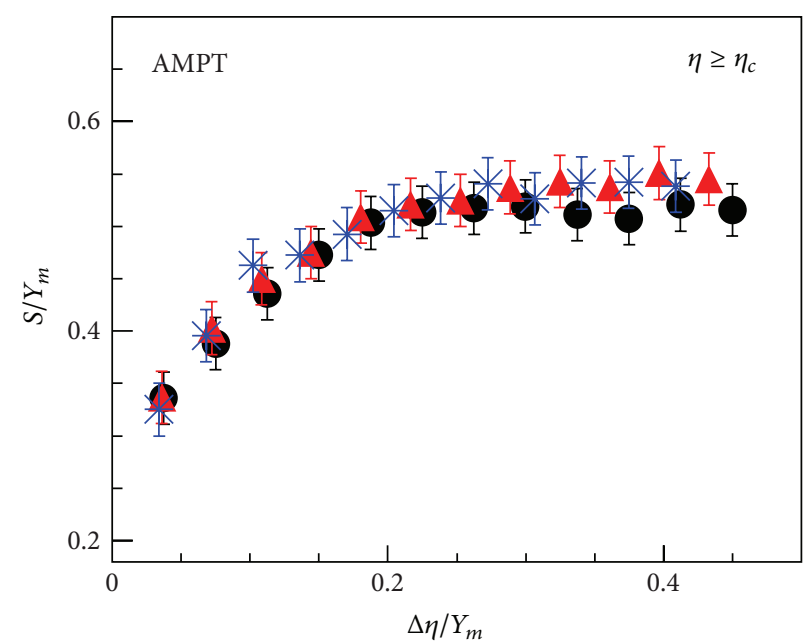

(b)

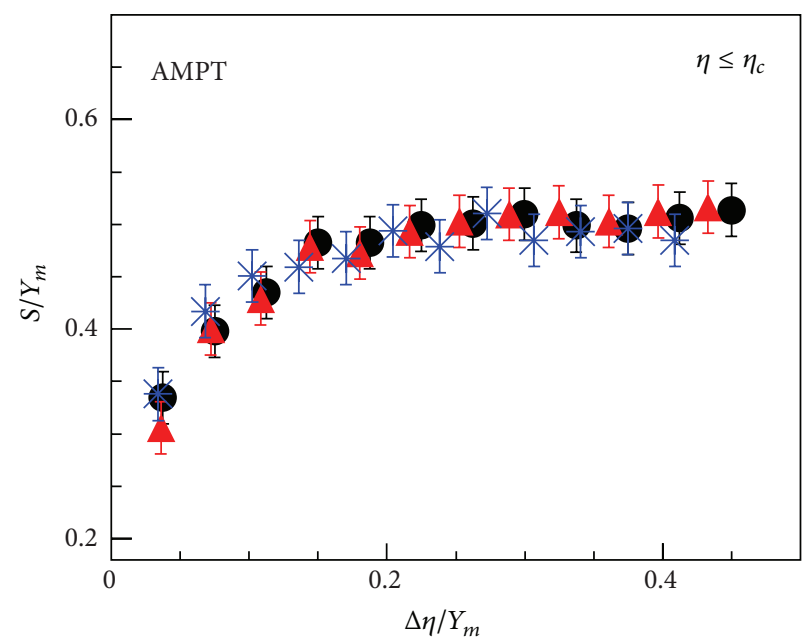

- $14.5 \mathrm{~A} \mathrm{GeV} / \mathrm{c}^{28} \mathrm{Si}-\mathrm{AgBr}$

$\triangle 60 \mathrm{~A} \mathrm{GeV} / \mathrm{c}^{16} \mathrm{O}-\mathrm{AgBr}$

* $200 \mathrm{~A} \mathrm{GeV} / \mathrm{c}^{16} \mathrm{O}-\mathrm{AgBr}$

(d)

FIgURE 6: Dependence $S / Y_{m}$ on $\Delta \eta / Y_{m}$ in the forward and backward regions for the real and AMPT events at the three incident energies.

from the available measurements of particle yields, spectra, and source size indicates that at the same energy density, QGP would be a high entropy state as compared to pion gas. It may be stressed that an increase in the entropy density, if observed at RHIC or LHC energies, might be taken as a signal of QGP formation.

\section{References}

[1] M. Rybezynski, "Energy dependence of fluctuations in central $\mathrm{Pb}+\mathrm{Pb}$ collisions from NA49 at the CERN SPS," Journal of Physics G, vol. 35, no. 10, Article ID 104091, 2008.

[2] E. V. Shuryak, "Quantum chromodynamics and the theory of superdense matter," Physics Reports C, vol. 61, no. 2, pp. 71-158, 1980.
[3] D. Ghosh, A. Deb, S. Bhattacharyya, and U. Datta, "Rapidity dependence of multiplicity fluctuations and correlations in high-energy nucleus-nucleus interactions," Pramana, vol. 77, no. 2, pp. 297-313, 2011.

[4] A. Bialas and W. Czyz, "Event by event analysis and entropy of multiparticle systems," Physical Review D, vol. 61, no. 7, Article ID 074021, 7 pages, 2000.

[5] A. Bialas and W. Czyz, "Measurement of entropy of a multiparticle system: a do-list," Acta Physica Polonica B, vol. 31, no. 3, pp. 687-692, 2000.

[6] A. Bialas and W. Czyz, "Renyi entropies are calculated for some multiparticle systems," Acta Physica Polonica B, vol. 31, no. 12, p. 2803, 2000.

[7] A. Bialas and W. Czyz, "Renyi entropies for bernoulli distributions," Acta Physica Polonica B, vol. 32, no. 10, p. 2793, 2001.

[8] P. A. Miller, S. Sarkar, and R. Zarum, "Quantum chaos: entropy signatures," Acta Physica Polonica B, vol. 29, no. 12, p. 3436, 1998. 
[9] M. R. Atayan, B. Yuting, E. A. de Wolf et al., "Entropy analysis in $\pi+p$ and $K+p$ collisions at $\sqrt{s}=22 \mathrm{GeV}$," Acta Physica Polonica B, vol. 36, no. 10, p. 2969, 2005.

[10] A. Bialas, W. Czyz, and J. Wosiek, "Studying thermodynamics in heavy ion collisions," Acta Physica Polonica B, vol. 30, no. 1, p. 107, 1999.

[11] K. Fialkowski and R. Wit, "Entropy in hadron-hadron collisions," Physical Review D, vol. 62, no. 11, Article ID 114016, 4 pages, 2000.

[12] A. Bialas, W. Czyz, and A. Ostruszka, "Renyi entropies in particle cascades," Acta Physica Polonica B, vol. 34, no. 1, p. 69, 2003.

[13] S. K. Ma, Statistical Mechanics, World Scientific, 1985.

[14] S. K. Ma, "Calculation of entropy from data of motion," Journal of Statistical Physics, vol. 26, no. 2, pp. 221-240, 1986.

[15] S. K. Ma and M. Payne, "Entropy of a spin-glass model with long-range interaction," Physical Review B, vol. 24, no. 7, pp. 3984-3990, 1981.

[16] V. Simak, M. Šumbera, and I. Zborovský, "Entropy in multiparticle production and ultimate multiplicity scaling," Physics Letters B, vol. 206, no. 1, pp. 159-162, 1988.

[17] T. Mizoguchi and M. Biajima, "Analyses of multiplicity distributions with $\eta_{c}$ and Bose-Einstein correlations at LHC by means of generalized Glauber-Lachs formula," The European Physical Journal C, vol. 70, no. 4, pp. 1061-1069, 2010.

[18] D. Ghosh, A. Mukhopadhyay, A. Ghosh et al., "Multiplicity characteristics of symmetric and asymmetric heavy-ion interactions at 4.5 A GeV/c," Europhysics Letters, vol. 11, no. 6, p. 535, 1990.

[19] D. Ghosh, A. Deb, P. K. Haldar et al., "Validity of the negative binomial multiplicity distribution in case of ultra-relativistic nucleus-nucleus interaction in different azimuthal bins," Europhysics Letters, vol. 65, no. 3, p. 311, 2004.

[20] D. Ghosh, A. Deb, S. Bhattacharyya et al., "Entropy scaling in high-energy nucleus-nucleus interactions," Journal of Physics $G$, vol. 38, no. 6, Article ID 065105, 2011.

[21] M. M. Khan, N. Ahmad, M. D. Azmi et al., "Evidence of entropy scaling in relativistic nucleus-nucleus collisions," International Journal of Modern Physics E, vol. 21, no. 7, Article ID 1250068, 8 pages, 2012.

[22] G. F. Bertsch, "Entropy and composite particle production," Nuclear Physics A, vol. 400, pp. 221-232, 1983.

[23] P. Seimens and J. Kapusta, "Evidence for a soft nuclear-matter equation of state," Physical Review Letters, vol. 43, no. 20, pp. 1486-1489, 1979.

[24] S. Pal and S. Pratt, "Entropy production at RHIC," Physics Letters $B$, vol. 578, no. 3-4, pp. 310-317, 2004.

[25] M. I. Adamovich, M. M. Aggarwal, Y. A. Alexandrov et al., "Produced particle multiplicity dependence on centrality in nucleus-nucleus collisions," Journal of Physics G, vol. 22, no. 10, p. 1469, 1996.

[26] M. I. Adamovich, M. M. Aggarwal, N. P. Andreeva et al., "Rapidity densities and their fluctuations in central $200 \mathrm{~A}$ $\mathrm{GeV}{ }^{32} \mathrm{~S}$ interactions with $\mathrm{Au}$ and $\mathrm{Ag}, \mathrm{Br}$ nuclei EMU01 collaboration," Physics Letters B, vol. 227, no. 2, pp. 285-290, 1989.

[27] M. I. Adamovich, M. M. Aggarwal, R. Arora et al., "Scaling properties of charged particle multiplicity distributions in oxygen induced emulsion interactions at 14.6, 60 and $200 \mathrm{~A}$ GeV,' Physics Letters B, vol. 223, no. 2, pp. 262-266, 1989.
[28] M. I. Adamovich, Y. A. Alexandrov, S. A. Asimov et al., "Multiplicities and rapidity densities in $200 \mathrm{~A} \mathrm{GeV}^{16} \mathrm{O}$ interactions with emulsion nuclei," Physics Letters B, vol. 201, no. 3, pp. 397402, 1988.

[29] C. F. Powell, P. H. Fowler, D. H. Perkins et al., The Study of Elementary Particles by Photographic Method, Pergamon Press, Oxford, UK, 1959.

[30] S. Ahmad, A. Ahmad, A. Chandra et al., "Forward-backward multiplicity fluctuations in $200 \mathrm{~A} \mathrm{GeV} / \mathrm{c}^{16} \mathrm{O}-\mathrm{AgBr}$ and ${ }^{32} \mathrm{~S}-\mathrm{AgBr}$ collisions," Physica Scripta, vol. 87, no. 4, Article ID 045201, 2013.

[31] S. Ahmad, A. R. Khan, M. Zafar, and M. Irfan, "On multifractality and multifractal specific heat in ion-ion collisions," Chaos, Solitons \& Fractals, vol. 42, no. 1, pp. 538-547, 2009.

[32] M. L. Cherry, A. Dabrowska, P. Deines-Jones et al., "Event-byevent analysis of high multiplicity $\mathrm{Pb}(158 \mathrm{GeV} /$ nucleon $)-\mathrm{Ag} / \mathrm{Br}$ collisions," Acta Physica Polonica B, vol. 29, no. 8, pp. 2129-2146, 1998.

[33] Z. W. Lin, C. M. Ko, B.-A. Li et al., "Multiphase transport model for relativistic heavy ion collisions," Physical Review C, vol. 72, no. 6, Article ID 064901, 29 pages, 2005.

[34] Y. M. Sinyukov and S. V. Akkelin, "Pion entropy and phasespace densities in A+A collisions," Acta Physica Hungarica A, vol. 22, no. 1-2, pp. 171-178, 2005.

[35] S. Ahmad, M. M. Khan, N. Ahmad, A. R. Khan, M. Zafar, and M. Irfan, "Erratic fluctuations in $14.5 \mathrm{~A} \mathrm{GeV} / \mathrm{c}^{28} \mathrm{Si}-\mathrm{AgBr}$ collisions," Acta Physica Hungarica A, vol. 25, no. 1, pp. 105-115, 2006.

[36] D. Ghosh, S. Sen, and J. Roy, "Maximum fluctuations of particle densities in narrow pseudorapidity space in highenergy interaction of hadrons with nuclei," Physical Review D, vol. 47, no. 3, pp. 1235-1238, 1993.

[37] H. R. Schmidt and J. Schukraft, "The physics of ultra-relativistic heavy-ion collisions," Journal of Physics G, vol. 19, no. 11, pp. 1705-1795, 1993.

[38] K. Wozniak, "Multiplicity fluctuations in $\mathrm{Au}+\mathrm{Au}$ collisions at RHIC, International Journal of Modern Physics E, vol. 16, p. 2187, 2007.

[39] P. Stainberg, "Forward-backward multiplicity correlations in $\sqrt{s_{N N}}=200 \mathrm{GeV} \mathrm{Au}+\mathrm{Au}$ collisions," Nuclear Physics A, vol. 774, pp. 631-634, 2006.

[40] B. Alver, B. B. Back, M. D. Baker et al., "System size dependence of cluster properties from two-particle angular correlations in $\mathrm{Cu}+\mathrm{Cu}$ and $\mathrm{Au}+\mathrm{Au}$ collisions at $\sqrt{s_{N N}}=200 \mathrm{GeV}$,' Physical Review C, vol. 81, no. 2, Article ID 024904, 9 pages, 2010.

[41] B. Alver, B. B. Back, M. D. Baker et al., "Cluster properties from two-particle angular correlations in $\mathrm{p}+\mathrm{p}$ collisions at $\sqrt{s}=200$ and $410 \mathrm{GeV}$," Physical Review C, vol. 75, no. 5, Article ID 054913, 8 pages, 2007.

[42] B. I. Abelev, M. M. Aggarwal, Z. Ahammed et al., "Growth of long range forward-backward multiplicity correlations with centrality in $\mathrm{Au}+\mathrm{Au}$ collisions at $\sqrt{s_{N N}}=200 \mathrm{GeV}$," Physical Review Letters, vol. 103, no. 17, Article ID 172301, 6 pages, 2009.

[43] V. P. Konchakovski, M. Hauer, G. Torrieri, M. I. Gorenstein, and E. L. Bratkovskaya4, "Forward-backward correlations in nucleus-nucleus collisions: baseline contributions from geometrical fluctuations," Physical Review C, vol. 79, no. 3, Article ID 034910, 6 pages, 2009.

[44] M. Skoby, "Forward-backward multiplicity correlations at STAR," Nuclear Physics A, vol. 854, no. 1, pp. 113-116, 2011.

[45] B. K. Rivastava, R. P. Scharenberg, and T. J. Tarnowsky, "Understanding the particle production mechanism with correlation 
studies using long and short range correlations," International Journal of Modern Physics B, vol. 16, p. 2210, 2007.

[46] B. K. Srivastava, "Fluctuations and correlations in STAR," European Physical Journal A, vol. 31, no. 4, pp. 862-867, 2007. 

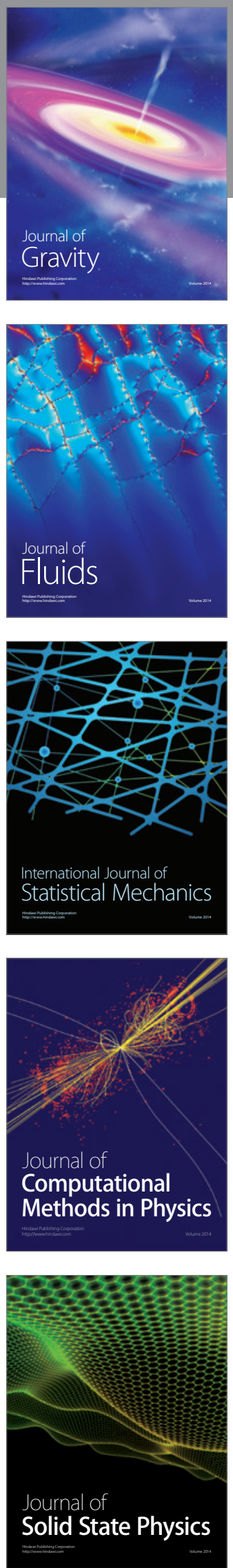

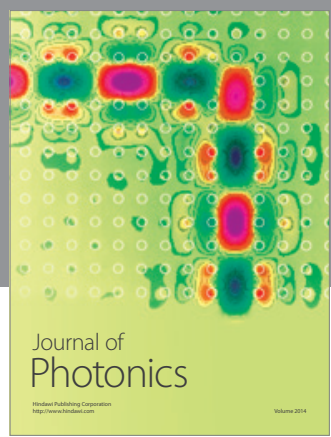

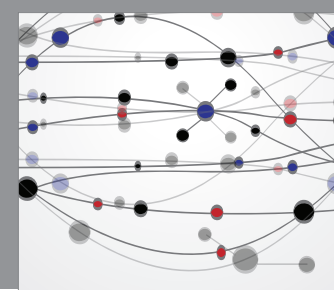

The Scientific World Journal

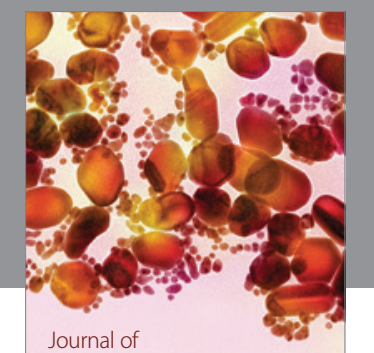

Soft Matter
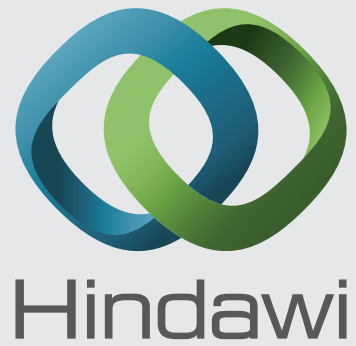

Submit your manuscripts at

http://www.hindawi.com
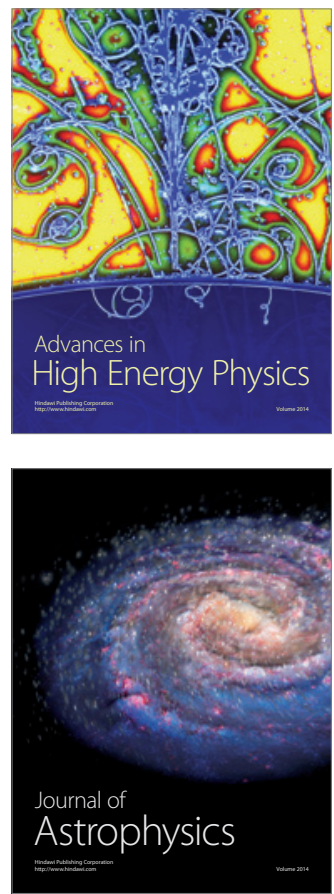
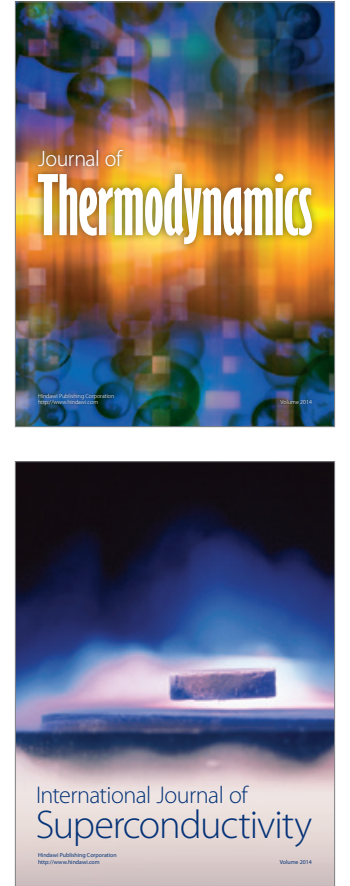
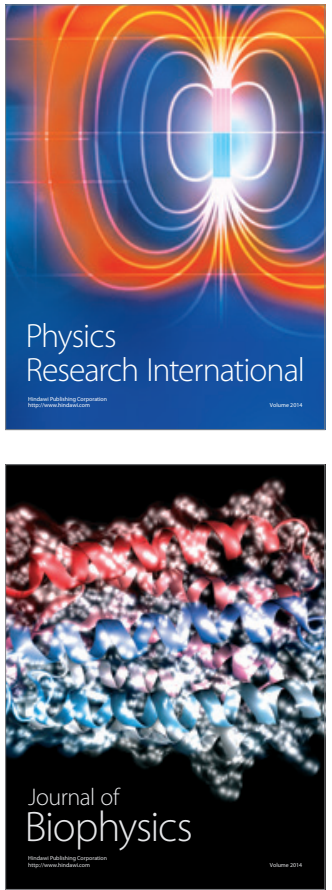
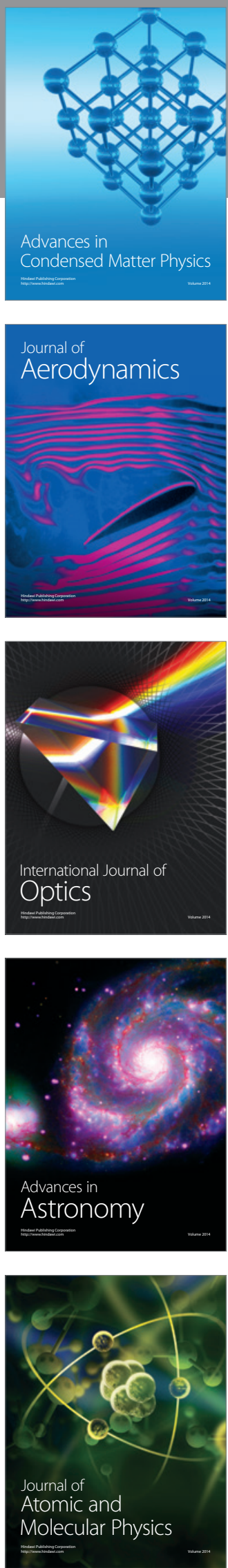